\title{
Modeling and Simulation for Dynamic Effect in 3D Virtual Scene System
}

\author{
Zhang Yue ${ }^{1, ~ a, ~ L v ~ H u i F a n g ~}{ }^{1, b}$ \\ ${ }^{1}$ Military Economics Academy, Wuhan, China \\ azhangyue_wuhan@sina.cn, ${ }^{b} 731027$ Ihf@163.com
}

Keywords: Virtual scene, DOF, Switch, Dynamic effect, Texture mapping

\begin{abstract}
Aiming at difficulty of modeling and simulation for dynamic effect in 3D virtual scene system, some methods combined with Multigen creator and Vega Prime are put forward. Firstly, rise-descend and rotation effect are simulated by adopting DOF node. Secondly, screen-show effect is realized with using switch node. Thirdly, some dynamic effects such as rolling wheel are simulated based on multiple textures mapping technology. Finally, object positioning dynamically based on coordinate transforming is proposed.
\end{abstract}

\section{Introduction}

Dynamic effects are mainly consists of wheel rolling, equipment operation, screen display changing applied technologies such as switch node and texture mapping in 3D virtual scene simulation system. Its applications are becoming increasingly widespread with the fidelity requirements growing higher than before. For the system requirement mentioned above, some common dynamic effects are modeled and simulated based on Multigen Creator and Vega Prime. Quite satisfactory simulation results are got in 3D virtual scene simulation system and it can provide benefit reference for other similar simulation system.

\section{Modeling and Simulation for Rise-Descend and Rotation Effect Based on DOF Node}

When modeling for rise-descend action owned by antenna, the most effective method is setting DOF (Degree of Freedom) node for its models in order to reducing development workload and enhancing the realism of the 3D model. The model object will has motion feature by setting DOF node. Above all, the hierarchy of freedom must be considered when DOF node is set for those complicated motion of model. The 3D model will act in accordance with logical pattern only by setting its parameters reasonably.

Taking the example of antenna in the course of setting up, the last section of antenna body will rise with the current section and its head section will rise with the nearest section. The influence of DOF will accumulate along with the direction of antenna axis in the course of antenna rising. So every section below should be the parent node for last section and DOF of child node will inherited from motion and restraint of parent node. In this way, the hierarchy of DOF for antenna node can be set as it is shown in Fig. 1.

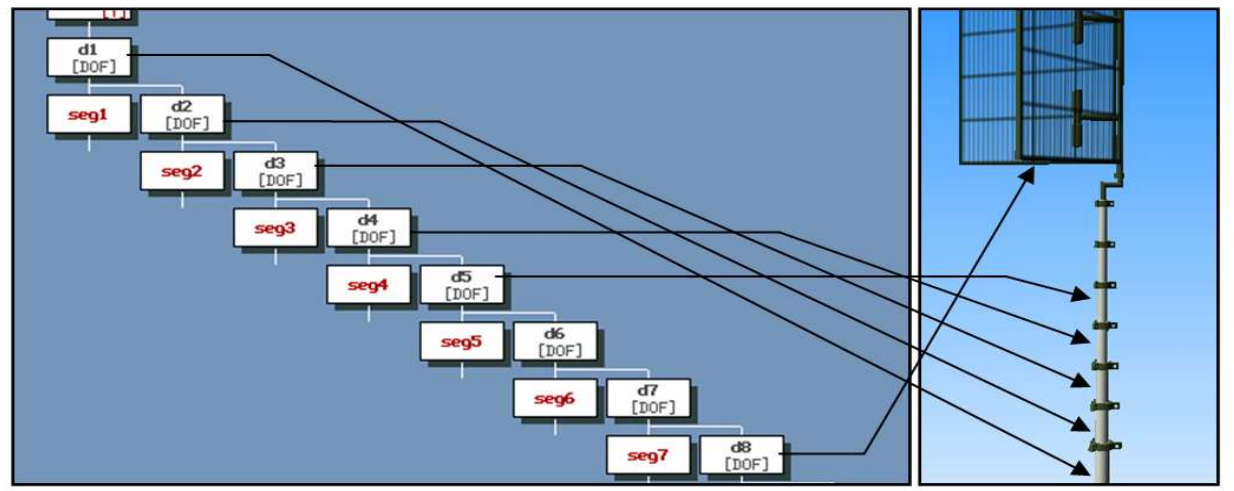

Fig. 1. The hierarchy of the DOF node for antenna 
Rise-descend of antenna and rotation of antenna head can be realized with setting its motion type of DOF node. Two different motion types including rise-descend and rotation in fixed axis direction will be implemented with DOF node. Its motion ranges are rise-descend height and rotation angle. The dynamic effect of rise-descend and rotation after setting its DOF nodes are shown as Fig. 2 and Fig. 3.
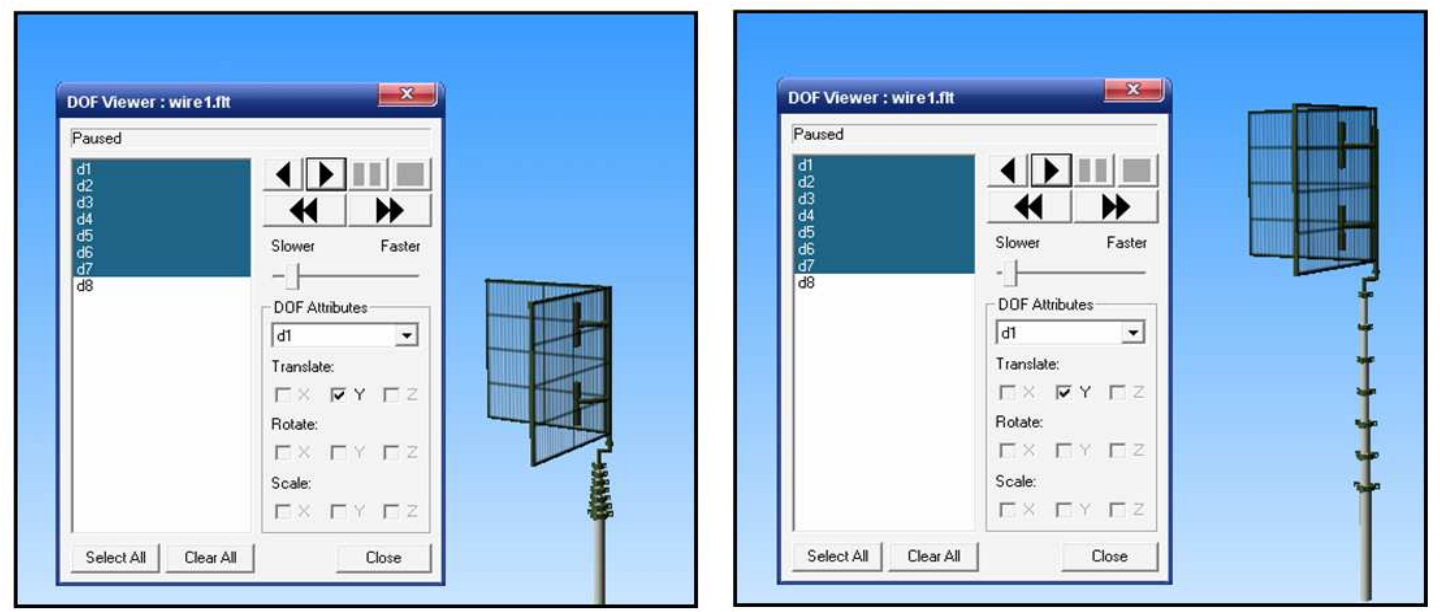

Fig. 2. The dynamic effect of lifting antenna
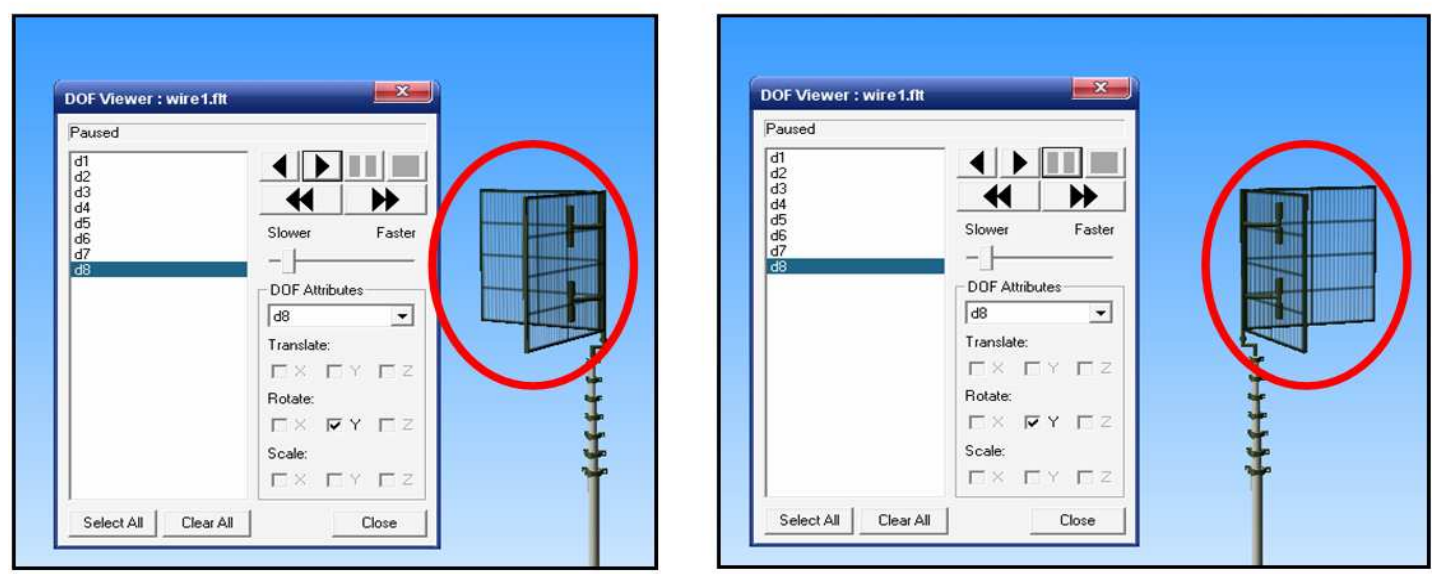

Fig. 3. The dynamic effect of rotating antenna head

\section{Modeling and Simulation for Screen-Show Effect Based on Switch Node}

Screen is an important interface for external information output of equipment. It is also an important channel for interaction between trainees and 3D scene simulation system. The content of screen-show reflects current operation and runtime status of equipment. For actual equipment, its operation will be translated into relevant data value after receiving input operation. Its output will be presented as a graph on the screen by resolving and computing with these data in system. In practice, we found that the screen-show effect is essentially a dynamic process, which a series of textured rectangular area switch in accordance with some specific rules. So screen-show effect can be simulated realized dynamically with applying switch node flexibility.

In the process of simulation realization, many child nodes of switch node will be correspond to many kinds of display interface. Each interface corresponds to a textured rectangular area and associates with a switch node. Each display status of switch node corresponds to a binary mask code and $0 / 1$ status of mask code determines whether the rectangular area is showing or hiding. Then all rectangular areas add together and their switch nodes will be child nodes of screen object. When an interface is need to be show in screen, each mask code of switch node will be assigned. So only rectangular areas corresponding to current interface will be shown and other rectangular areas will be hidden, and screen-show switching effect is simulated in this way. 
Taking the example of some radio model, its screen has many kinds of display interface such as frequency configuration, speed setting, and so on. These interfaces will be associated with switch nodes and their mask codes will be changed with display status. The dynamic screen-show effect is shown as Fig. 4.
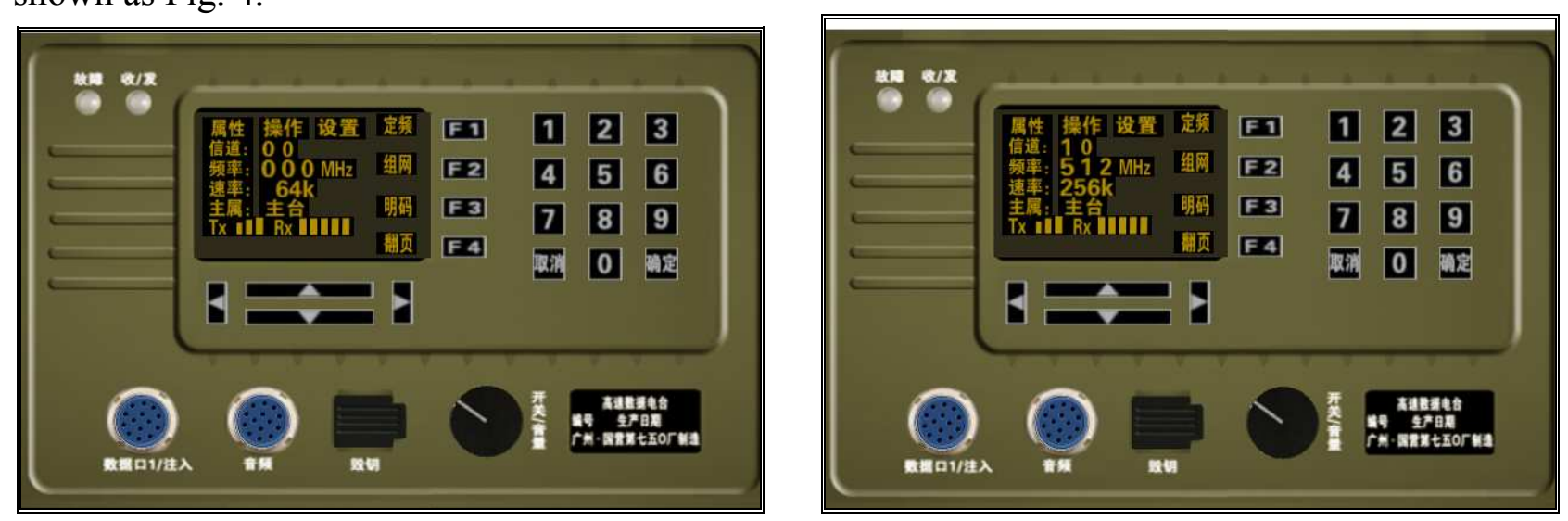

Fig. 4. The dynamic screen-show effect of radio applying switch node

\section{Modeling and Simulation for Rolling Wheel Effect Based on Multiple Texture Mapping}

For those dynamic effects consists of waving flag, flashing neon lights and rolling wheel, two different kinds of methods are designed. These methods are called "multiple faces and multiple textures" and "single face and multiple textures". The former method creates multiple identical faces and maps different textures sequence for these faces. Once the time interval is set, multiple textured faces will switch recurrently according to designed time interval and smooth 3D dynamic effect will be generated. Though added model faces and extra burden will be brought to graphic system in former method, it fits with large numbers of textures occasions due to no limitation for the mapping texture.

The main characteristics of latter method are adopting texture binding technology and only one face need to be created to map multiple different textures sequence. Then dynamic 3D effect will be presented after corresponding time interval is set and the above textures are played dynamically in that face. Though lesser faces are used to modeling in latter method, it has limits only on texture number mapping on this face. So it is relatively suitable for simulation to dynamic 3D scene such as flowing river surface, rolling wheel, and so on. The dynamic effect of rolling wheel for some armored vehicle is shown as Fig. 5.

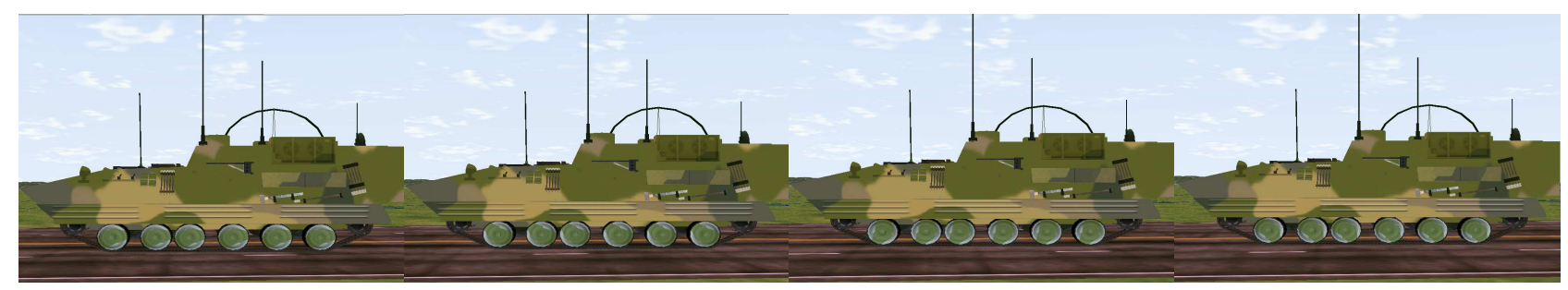

Fig. 5. The dynamic effect of rolling wheel for some armored vehicle

\section{Simulation for Positioning Object Dynamically Based on Coordinate Transforming}

In some 3D scene simulation system, object need to be moved from one place to other place, and the object must be positioned in the 3D scene accurately. For this kind of positioning object dynamically, DOF technology must be adopted and six position-orientation parameters must be computed dynamically. Essentially, it is a coordinate transforming problem, which transforms world coordinate system to local coordinate system. If local coordinate of object is $(0,0,0)$, the coordinate of local coordinate origin in world system is $(x, y, z)$, the orientation vector of local system to world system is $(h, p, r)$, the world coordinate of target position is $(p x, p y, p z)$, the local coordinate of target position is $\left(x^{1}, y^{1}, z^{1}\right)$. So the coordinate transforming equation is described as follow. 


$$
\left(\begin{array}{ll}
x^{\prime}, & y^{\prime}
\end{array} z^{\prime}\right)=(p x-x, \quad p y-y, \quad p z-z) \times\left|\begin{array}{ccc}
\cosh \cos r-\sinh \sin p \sin r & -\sinh \cos p & \cosh \sin r+\sinh \sin p \cos r \\
\sinh \cos r+\cosh \sin p \sin r & \cosh \cos p & \sinh \sin r-\cosh \sin p \cos r \\
-\cos p \sin r & \sin p & \cos p \cos r
\end{array}\right|
$$

With this method, the whole erecting process of antenna in some armored vehicle is simulated in view of coordinate transforming. The simulation examples are shown as Fig. 6.
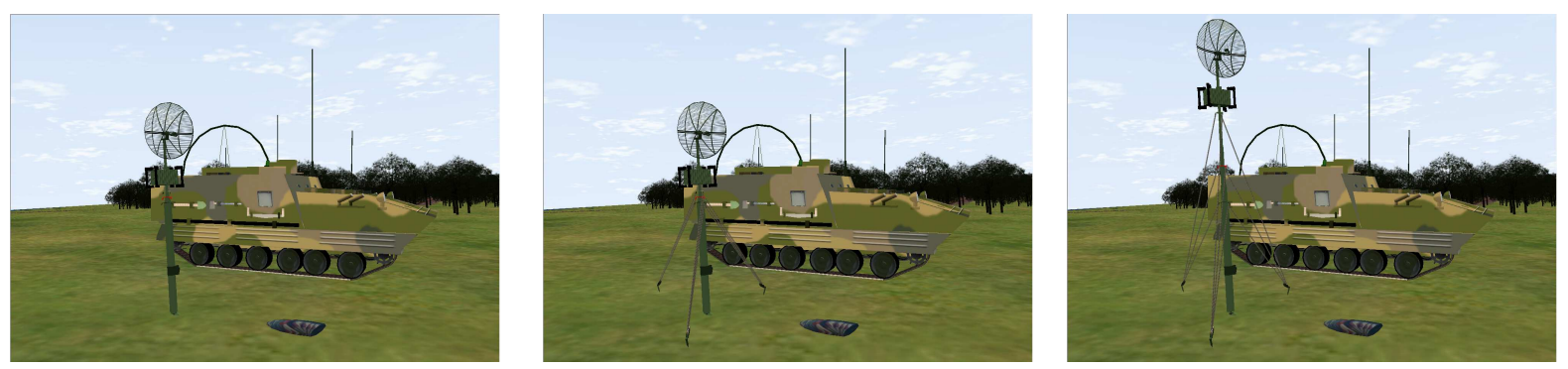

Fig. 6. The simulation example of erecting antenna in some armored vehicle

\section{Conclusion}

The fidelity of dynamic effec is a direct sign to measure 3D virtual scene system. Considering most of these effects have same realization technologies, some methods for implementing them including rise-descend, rotation, screen-show and rolling wheel are detailed. Some given simulation examples have shown its feasibility and its implementation details were also presented. It can provide foundation for realization similar 3D virtual scene simulation system.

\section{References}

[1] Gang Chen and Jun Yang, Research on 3D Equipment Training Simulator, 2010 2nd International Conference on Computer Engineering and Technology, vol. 7, pp. 593-597, 2010.

[2] LIU Hang, WANG Chun-shui and WANG Ji-zhong, A Virtual Operation Training System Based on Visual Scenery Simulation Technology, Command Control \& Simulation, vol. 29, pp. 79-82, 2007.

[3] HE Shu-you, REN Hai-peng and Gao Ang, Design and Realization of The Special Effect on Fly Track for Certain Missile, Shipboard Electronic Countermeasure, vol. 30, pp. 89-91, 2007.

[4] DUAN Chunmei, ZHANG Yan and ZHAN Shouyi, Research and Design of Extended Vega FX Module, Computer Engineering, vol. 32, pp. 271-273, 2006.

[5] ZHENG Lin, LI Jian, LIU Xiang-kai and WANG Cheng-wu, Design and Development of Scene Simulation Platform, Journal of System Simulation, vol. 22, pp. 1182-1188,1194, 2010.

[6] LI Yachen, JIANG Hongliu, XIONG Hailin, LUO Yongfeng and YAN Peng, 3D Earth Modeling in Visual Simulation, vol. 33, pp. 225-227, 2007.

[7] WEI Xu-guan, ZHU Li-feng, HUANG-Hao and WANG Rong-chang, Research of the 3D scene simulation in battlefield environment and key algorithm of a watercraft simulation training system, Ship Science and Technology, vol. 30, pp. 119-124, 2008.

[8] WANG Hai-yang and GAO Qin-he, Study of Visual Simulation System for Erecting Process of Large Mechanism, Computer Simulation, vol. 24, pp. 184-186, 2007. 